\title{
Platelet factor 4 is a biomarker for lymphatic-promoted disorders
}

\author{
Wanshu Ma, ${ }^{1}$ Hyea Jin Gil, ${ }^{1}$ Noelia Escobedo, ${ }^{1}$ Alberto Benito-Martín, ${ }^{2}$ Pilar Ximénez-Embún, ${ }^{3}$ \\ Javier Muñoz, ${ }^{3}$ Héctor Peinado, ${ }^{4}$ Stanley C. Rockson, ${ }^{5}$ and Guillermo Oliver ${ }^{1}$ \\ 'Center for Vascular and Developmental Biology, Feinberg Cardiovascular and Renal Research Institute, Northwestern \\ University, Chicago, Illinois, USA. ${ }^{2}$ Children's Cancer \& Blood Foundation Laboratories, Departments of Pediatrics and Cell \\ and Developmental Biology, Weill Cornell Medicine, New York, USA. ${ }^{3}$ Proteomics Unit - ProteoRed-ISCIII, Spanish National \\ Cancer Research Centre, Madrid, Spain. ${ }^{4}$ Microenvironment \& Metastasis Group, Molecular Oncology Program, Spanish \\ National Cancer Research Center, Madrid, Spain. ${ }^{5}$ Division of Cardiovascular Medicine, Center for Lymphatic and Venous \\ Disorders, Stanford University School of Medicine, Stanford, California, USA.
}

Genetic or acquired defects of the lymphatic vasculature often result in disfiguring, disabling, and, occasionally, life-threatening clinical consequences. Advanced forms of lymphedema are readily diagnosed clinically, but more subtle presentations often require invasive imaging or other technologies for a conclusive diagnosis. On the other hand, lipedema, a chronic lymphatic microvascular disease with pathological accumulation of subcutaneous adipose tissue, is often misdiagnosed as obesity or lymphedema; currently there are no biomarkers or imaging criteria available for a conclusive diagnosis. Recent evidence suggests that otherwise-asymptomatic defective lymphatic vasculature likely contributes to an array of other pathologies, including obesity, inflammatory bowel disease, and neurological disorders. Accordingly, identification of biomarkers of lymphatic malfunction will provide a valuable resource for the diagnosis and clinical differentiation of lymphedema, lipedema, obesity, and other potential lymphatic pathologies. In this paper, we profiled and compared blood plasma exosomes isolated from mouse models and from human subjects with and without symptomatic lymphatic pathologies. We identified platelet factor 4 (PF4/CXCL4) as a biomarker that could be used to diagnose lymphatic vasculature dysfunction. Furthermore, we determined that PF4 levels in circulating blood plasma exosomes were also elevated in patients with lipedema, supporting current claims arguing that at least some of the underlying attributes of this disease are also the consequence of lymphatic defects.

Authorship note: WM and HJC contributed equally to this work.

Conflict of interest: The authors have declared that no conflict of interest exists

Copyright: (c) 2020, American Society for Clinical Investigation.

Submitted: November 19, 2019

Accepted: June 3, 2020

Published: June 11, 2020

Reference information: JCl Insight. 2020;5(13):e135109.

https://doi.org/10.1172/jci.

insight.135109.

\section{Introduction}

The lymphatic vasculature is a network of thin-walled initial lymphatic capillaries and larger collecting vessels covered by a continuous layer of endothelial cells providing a unidirectional conduit for filtered tissue interstitial fluids, metabolites, macromolecules, and cells toward the central venous circulation. Its principal function is to maintain fluid homeostasis by removing the protein-enriched fluids from the extracellular space and returning them, in the form of lymph, to the bloodstream (1). Lymphatics are also important for lipid transport and immune cell trafficking, among other functions.

One of the main disorders that ensue from malfunction of the lymphatic vasculature is lymphedema, a disfiguring, disabling, and occasionally, life-threatening clinical condition characterized by the localized interstitial accumulation of protein-rich fluid, thereby promoting tissue edema for which, at present, treatment options are few and have limited efficacy (2). This disease affects millions worldwide and most commonly entails swelling of the extremities, tissue fibrosis, susceptibility to infections, and accumulation of subcutaneous fat $(2,3)$. Lymphedema can result from either primary or acquired (secondary) disorders. Primary lymphedema is the consequence of genetic defects that affect the formation and normal function of the lymphatic vasculature and most commonly manifests during infancy, childhood, or adolescence $(2,4)$. Secondary lymphedema is the more common presentation and is caused by lymphatic trauma sustained after surgery, radiation therapy, infection, or trauma (2-4). In general, overt lymphedema can be diagnosed based on the clinical context and the physical examination; however, more precise staging and characterization require imaging protocols that are often invasive. 
A direct correlation and mechanistic relationship between the lymphatic vasculature and the adipose compartment have recently been recognized in patients with lymphatic disorders. Abnormal subcutaneous fat accumulation in the affected edematous regions in patients with secondary lymphedema is the inescapable consequence of sustained defective lymphatic drainage. Analysis of patients has also shown that malformation of cutaneous lymphatics causes bilateral fat accumulation in the thigh and buttock (5-7), a phenotype that worsens during puberty, while dermal lipid accumulation occurs in patients with idiopathic lymphedema (8, 9). Although physiotherapy and use of compression garments do limit interstitial fluid accumulation, at present there are limited options for the treatment of these more advanced manifestations of the disease.

Lipedema is a common, chronic lymphovascular disease (10-13) characterized by bilateral, symmetrical swelling in the extremities because of the deposition of abnormal subcutaneous adipose tissue (10, 11, 14). Lipedema, often misdiagnosed as obesity or lymphedema $(10,11,15-17)$, occurs almost exclusively in females and likely has a genetic component because a positive family history is common. Nevertheless, in contrast to lymphedema, overt interstitial edema is not observed in lipedema, and the swelling due to adipose hypertrophy occurs in a distinctly symmetrical pattern (16). Early studies by Bilancini et al. demonstrated that lipedema is consistently associated with functional alterations of the lymphatic vasculature (11). Using dynamic imaging, they showed that patients suffering from lipedema have an abnormal lymphoscintigraphic pattern, with a slowing of the lymphatic flow similar to the alterations found in patients with lymphedema (11). Despite these insights, lipedema is frequently misdiagnosed as obesity or lymphedema, and the pathogenesis and molecular mechanisms of this disease are still very poorly understood. Nevertheless, lipedema appears to be an adipose disorder with an apparent contribution of lymphatic malfunction. Whether those lymphatic alterations are partially responsible for the disease, or are secondary to the related obesity features, is not yet known. Unfortunately, even with focused morphological analysis, lipedema is not easy to differentiate from obesity; clinicians often lack familiarity with this condition, distinct clinical imaging attributes have not been identified, there are no known biomarkers for the disease, and conclusive mechanistic evidence supporting the proposal that lymphatic defects contribute to the disease is still lacking.

More recently, the functional roles of the lymphatic vasculature have broadened. New evidence suggests that asymptomatic defective or leaky lymphatic vessels could be responsible for certain forms of obesity (18, 19), inflammatory bowel disease/Crohn's disease, glaucoma, and some forms of neurological pathology (for a review, see ref. 20). Thus, identification of easily accessible, reliable biomarkers of lymphatic malfunction would be a valuable resource not only to assist in the conclusive diagnosis of lymphedema but also to facilitate the differential diagnosis among subjects with lymphedema, lipedema, and obesity. Furthermore, the identification of such biomarkers could eventually also help identify and diagnose subtle, asymptomatic lymphatic alterations that might contribute to some of the aforementioned disorders. Accordingly, we profiled and compared circulating exosomes isolated from blood plasma from animal models and from patients with and without documented lymphatic pathologies. Exosomes are small vesicles (30-100 $\mathrm{nm}$ in diameter) of endocytic origin secreted by most cells (including endothelial cells) (21-24). These extracellular vesicles contain cell type-specific proteins and genetic materials, including mRNAs, miRNAs, and DNA. They can also exert a functional influence once taken up by recipient cells, therefore representing novel mediators of intercellular communication (25-30). Exosomes are emerging biomarkers of various types of diseases (21).

In this investigation, we performed mass spectrometry (MS) analysis and compared exosome proteomic signatures in normal, obese, and lymphatic defective mouse models. A similar approach was used with plasma exosomes obtained from patients with various lymphatic disorders and with lipedema and from obese and nonobese individuals without clinically overt lymphatic dysfunction. We report on the identification of platelet factor 4 (PF4) as a plasma-circulating exosomal signature protein that could be used as a potentially novel biomarker in the clinical setting to diagnose lymphatic vasculature dysfunction and to distinguish these disorders from obesity not promoted by lymphatic disorders. Furthermore, we also found that PF4 levels were increased in circulating exosomes from patients with lipedema, a result that supports the prevailing hypothesis that the pathogenesis of this disease is, at least in part, lymphatic. However, exosomal PF4 levels were not associated with increased body weight, either in individuals with normal lymphatics or in those with lymphatic disorders.

\section{Results}

Exosome profiling in a mouse model of lymphatic malfunction. In an initial approach to determine whether blood plasma-derived exosomes might be used to identify lymphatic vascular defects, we first used available mouse models. We have previously reported that haploinsufficiency of Prox 1 in mice results in 


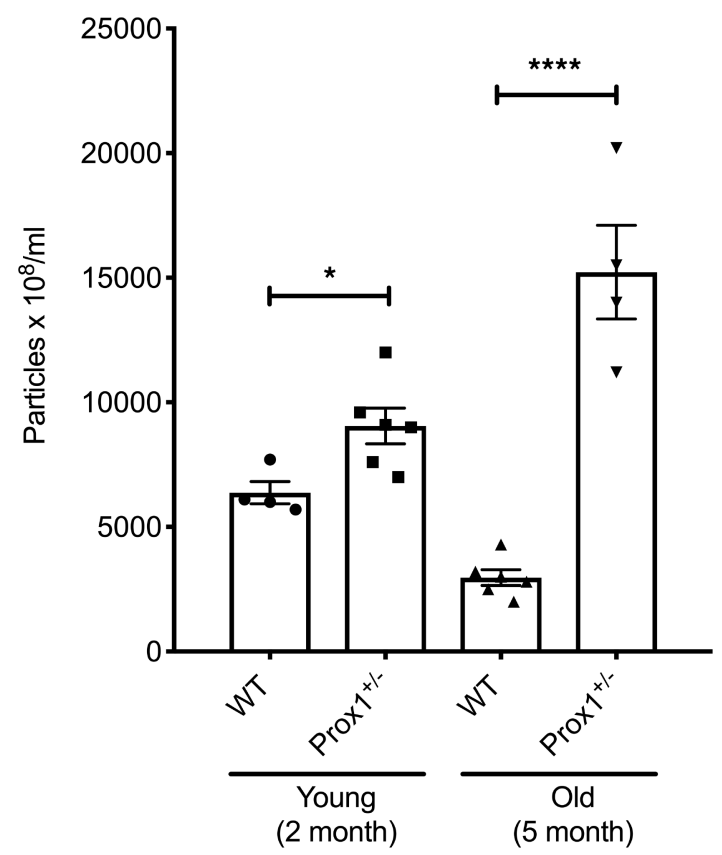

Figure 1. Characterization of plasma exosomes from young and old Prox $\mathbf{1}^{+/-}$mice. Exosome particle concentration is compared between young and old WT and $\operatorname{Prox}^{7^{+-}-}$mice $(N=4-6)$. Data represent mean value \pm standard error of the mean (SEM), and statistical analyses were performed by unpaired Student's $t$ test. ${ }^{*} P<0.05,{ }^{* * *} P<0.0001$.

morphological and functional alterations in the lymphatic vasculature that are associated with edema at midgestation and with obesity in adult animals (18). Detailed characterization of the lymphatic vasculature of E14.5 Prox $^{+/-}$embryos showed that embryos displayed edema, indicating lymphatic dysfunction, but this phenotype resolved before birth (18). Detailed characterization of the lymphatic vasculature of E16.5 Prox $^{+/-}$embryos and adult Prox $1^{+/-}$mice revealed mispatterning of the lymphatic vasculature; the most severely affected lymphatics were those of the intestine and mesentery, which were chyle filled and ruptured $(18,19)$. A low percentage of Prox $^{+/-}$mice survived to adulthood and became significantly heavier than WT littermates at approximately 4 months of age, a consequence of the subtle leakage of lymph/chyle that promotes visceral accumulation of fat, leading to obesity $(18,19)$. Accordingly, we compared the protein profile of plasma-circulating exosomes from young, nonobese ( $<3$ months) and older, obese (>5 months) Prox $1^{+/-}$mice; WT littermates; and $o b / o b$ mice (leptin receptor mutants) (31-33) that are severely obese but have a normal lymphatic vasculature (our unpublished data) (for each model we used mice of both sexes). We reasoned that by comparing those groups we should be able to identify biomarkers capable of distinguishing lymphatic malfunction (Prox ${ }^{+/-}$mice) from non-lymphatic-promoted obesity (i.e., $o b / o b$ mice) and from WT mice.

To isolate exosomes, terminal bleeding was performed, and blood was collected by cardiac puncture. Circulating exosomes were purified from the isolated plasma using standard protocols (see Methods for more information), and their presence and particle size were confirmed by Nanosight (34) and by electron microscopy (data not shown). Consistently, we found that in $\mathrm{ProxI}^{1^{+-}}$mice, the number of exosomes was higher than their age-matched littermate controls, either before or after the onset of obesity (Figure 1). Next, exosomes were subjected to MS to identify their protein cargo components. Due to the low survival rate of $\mathrm{Proxl}^{+/-}$mice and the low plasma volume, the exosome yield was low. Therefore, for the MS analysis, plasma samples of animals with the same genotype were pooled. We initially compared the proteomic signature of young (lean 3-month-old) and old (obese 5-month-old) Prox ${ }^{+/-}$mice and age-matched WT littermates using a fold change cutoff $>0.5$ or $<-0.5$ to identify the early changes that persist as disease develops. Using that criterion, 70 proteins were upregulated in both young and old Prox $1^{+/-}$mice (Figure $2 \mathrm{~A}$ ) and 36 were downregulated (Figure 2B). Important for the findings described below using the human samples, among the upregulated ones was PF4 (Figure 2A). Pathway enrichment and protein-protein interaction network analysis (Kyoto Encyclopedia of Genes and Genomes, KEGG) revealed that the upregulated proteins were mainly enriched in complement and coagulation cascades and systemic lupus erythematosus 
A
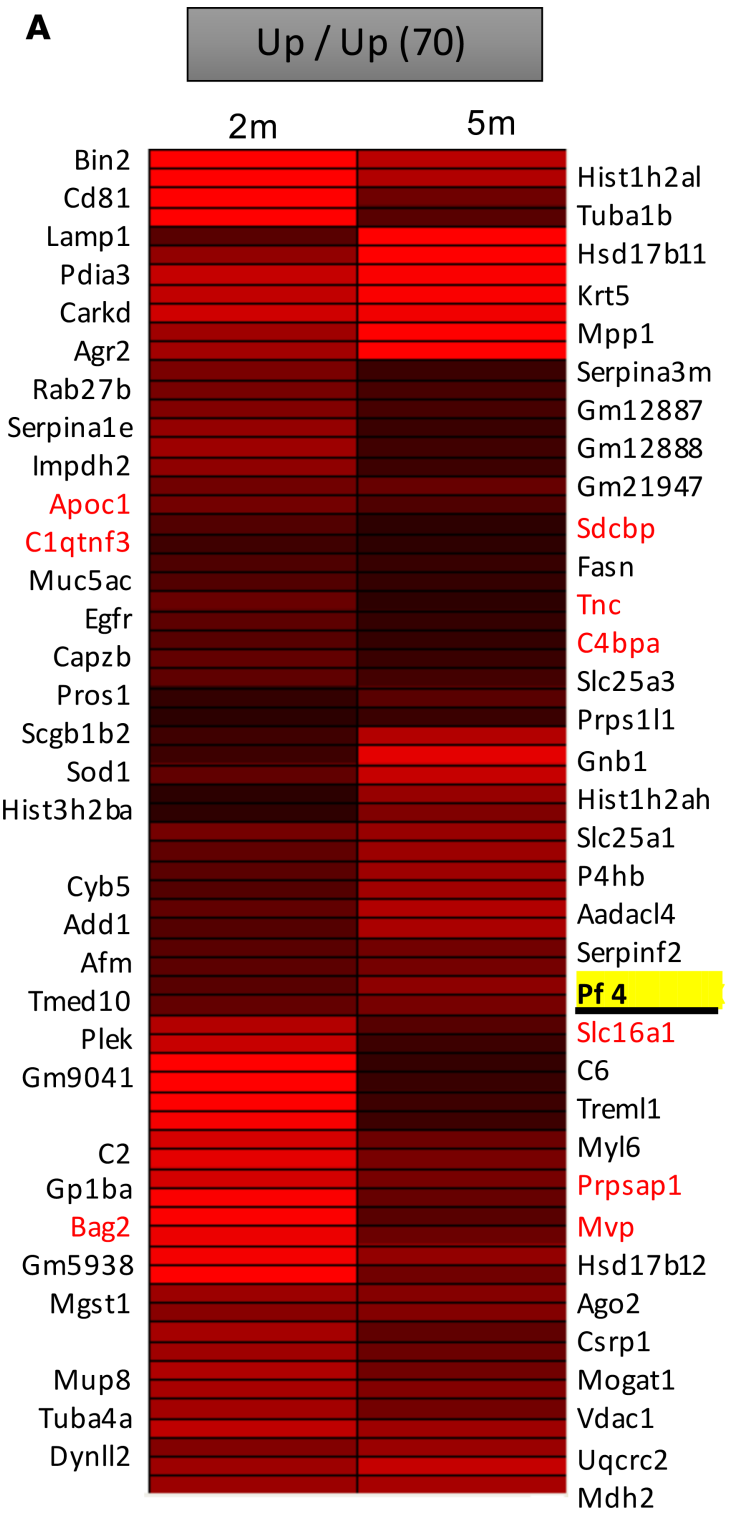

B

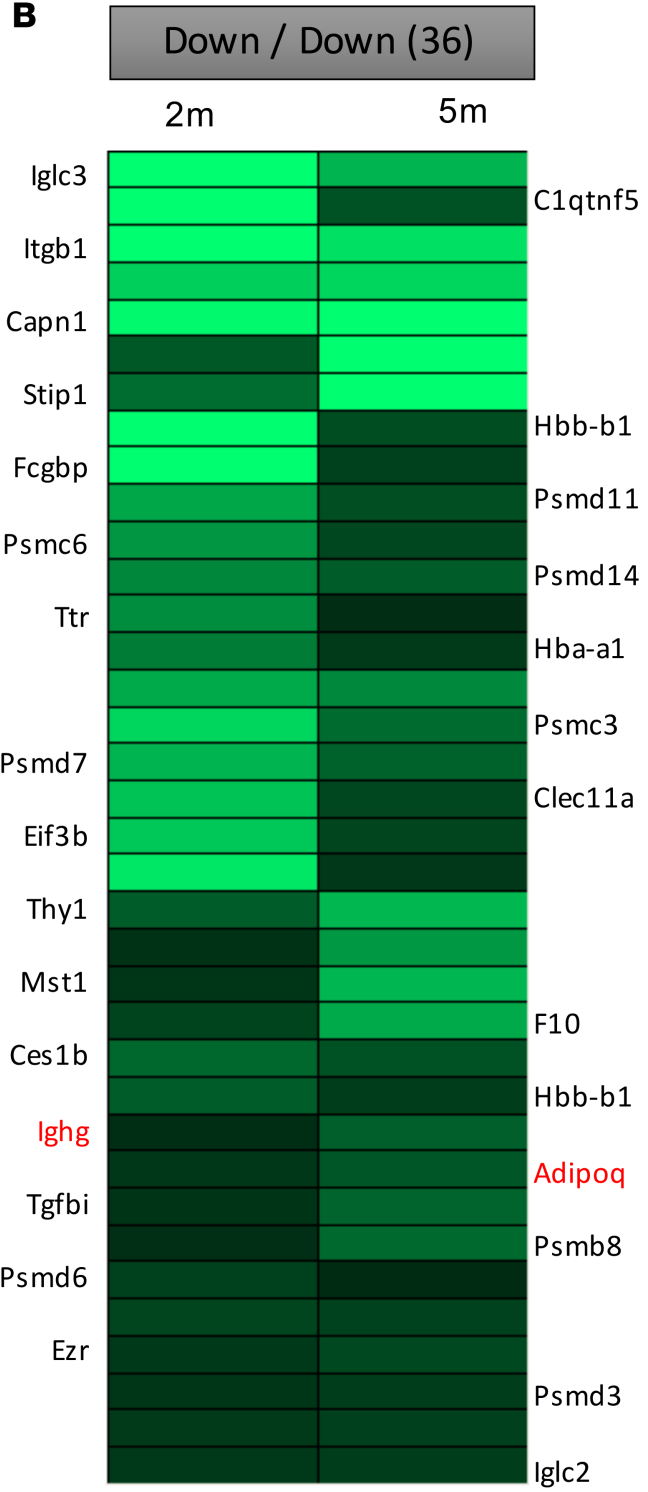

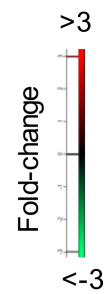

Figure 2. Protein signatures in plasma exosomes from young and old Prox $\mathbf{1}^{+/-}$mice. Proteins that are both increased (A) or decreased (B) in young and old Prox $1^{+/-}$mice were compared with age-matched WT mice. Gene name in red highlights the common changes in ob/ob mice. $(N=4-6$. $)$

pathways (Table 1); in contrast, the downregulated ones were identified mainly in proteasome, EpsteinBarr virus infection, and leukocyte transendothelial migration pathways (Table 2).

We then performed a similar MS analysis using pooled plasma from $o b / o b$ and WT mice. Among the 479 proteins, 187 were increased and 75 were decreased in the ob/ob group (Figure 3A). To exclude proteins related to obesity, we then compared the Prox ${ }^{+/-}$mice data set with the ones from WT and $o b / o b$ mice. We identified 9 upregulated proteins and 2 downregulated proteins common to $P r o x 1^{+/-}$and $o b / o b$ mice and narrowed the lymphatic signature in $\mathrm{ProxI}^{+/-}$to 61 upregulated and 34 downregulated proteins (Figure 3, B and C).

Isolation and characterization of exosomes from patients with lymphedema. To further validate and expand the animal model results described above, we next performed a similar analysis with plasma-circulating exosomes isolated from patients with lymphatic dysfunction and from normal subjects. To do this, we performed an initial pilot experiment; although the pilot study included a relatively limited number of subjects, the patient cohorts were generally well matched by demographic variables (Table 3). The studied cohorts included lean and obese healthy subjects without overt lymphatic dysfunction and patients with lymphatic disorders, including lean and obese subjects with secondary lymphedema, lean and obese subjects with lymphovascular disease, and lean and obese subjects with 
Table 1. KEGG pathway analysis of increased exosomal proteins in young and old Prox1+/- mice compared with age-matched WT mice

\begin{tabular}{|c|c|c|c|}
\hline Pathway & Description & Count in gene set & False discovery rate \\
\hline mmu04610 & Complement and coagulation cascades & 6 of 88 & 4.84E-05 \\
\hline mmu05322 & Systemic lupus erythematosus & 4 of 92 & 0.0123 \\
\hline
\end{tabular}

lipedema. As expected, all the lymphatic disease cohorts were female predominated (Table 3). Also, as anticipated, the category of lymphovascular disease, which reflects developmental and genetic diseases, was characterized by a significantly $(P<0.01)$ younger mean age.

Initially, we focused on the molecular differences between normal individuals and lymphatic disorder patients and therefore did not segregate individuals by BMI. Following exosome purification and MS analysis, we profiled 4 samples pooled from 8 normal subjects without overt lymphatic dysfunction, 8 pooled samples from 15 patients with secondary lymphedema, 3 samples from 3 patients with lymphovascular disease, and 8 samples from 8 patients with lipedema. Proteins with a $P$ value less than 0.1 and $\log _{2}$ ratio greater than 1 or less than -1 were considered differentially regulated. From this analysis, we identified 13 increased and 14 decreased proteins in patients with secondary lymphedema (Table 4 and Table 5), 38 increased and 55 decreased proteins in patients with lymphovascular disease (Table 6 and Table 7; only the top 50 decreased proteins are shown because of space limits), and 19 increased and 35 decreased proteins in patients with lipedema (Table 8 and Table 9). Of interest, and as shown in Table 4, Table 6 , and Table 8 , among this list of upregulated proteins, PF4 was the only one whose levels were elevated (when compared with normal controls) in samples from patients with secondary lymphedema, lymphovascular disease (including primary lymphedema), and lipedema and in Prox $^{+/-}$mice (Figure 2A). PF4 is a protein released from platelets and is known to be able to inhibit angiogenesis and to promote innate immune responses, making this protein an interesting target for inflammation. PF4 bound to surface glycosaminoglycans on platelets, monocytes, and endothelial cells is also an immunogenic target in prothrombotic disorders. The concentration of PF4 in serum after platelet activation is a thousand-fold higher than in plasma (35-38).

Next, we decided to further validate these initial results using a human PF4 ELISA. The exosome protein cargo from 12 normal subjects, 37 patients with lymphedema, 11 patients with lymphovascular disease, and 15 patients with lipedema was analyzed (protein content was normalized for each sample). This ELISA analysis validated the MS results described above, as PF4 levels were elevated in all patients except 1 (Figure 4A); as determined by Grubb's test (39), this single nonlymphedema obese patient with very high PF4 levels was an outlier with a history of inflammatory bowel disease (IBD); therefore, it was removed from this graph (Figure 4A). Although the pathophysiology of IBD remains unknown, alterations in the intestinal lymphatics are becoming accepted features of IBD, particularly in subjects with Crohn's disease (40-42). To evaluate the diagnostic power of PF4 for lymphatic alterations, a receiver operating characteristic (ROC) curve analysis was performed. As shown in Figure 4B, the AUCs were 0.80 (95\% CI: 0.67 to 0.93 ), 0.86 (95\% CI: 0.70 to 1.00 ), and 0.95 (95\% CI: 0.99 to 1.00) for patients with secondary lymphedema, lymphovascular disease, and lipedema, respectively. At the corresponding optimal cutoff values, the sensitivities and specificities of PF4 to predict secondary lymphedema reached $59.46 \%$ and $90.91 \%$, for lymphovascular disease reached $70.00 \%$ and $90.91 \%$, and for lipedema reached $86.67 \%$ and $90.91 \%$, respectively.

Table 2. KEGG pathway analysis of decreased exosomal proteins in young and old Prox1+- mice compared with age-matched WT mice

\begin{tabular}{|c|c|c|c|}
\hline Pathway & Description & Count in gene set & False discovery rate \\
\hline mmu03050 & Proteasome & 8 of 45 & 4.13E-14 \\
\hline mmu05169 & Epstein-Barr virus infection & 7 of 205 & 8.27E-08 \\
\hline mmu04670 & Leukocyte transendothelial migration & 3 of 115 & 0.0051 \\
\hline
\end{tabular}



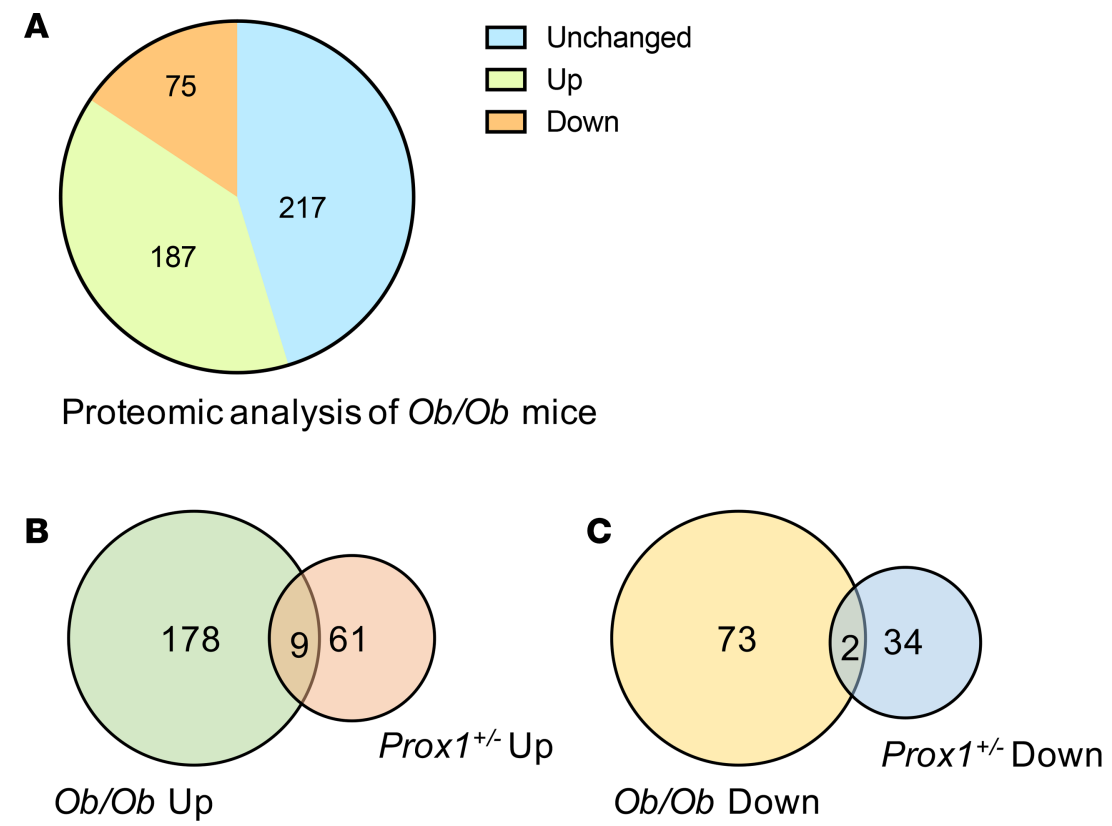

Figure 3. Proteomic analysis of plasma exosomes from ob/ob mice compared with WT controls. (A) Pie chart shows upregulated and downregulated protein changes in ob/ob mice compared with WT controls. $(N=3$.) (B-C) Venn diagram shows the common and unique proteins in Prox1 ${ }^{+/-}$compared with ob/ob mice. The common proteins are presented in red fonts in Figure 2, A and B.

To eliminate the likelihood that comorbidities might be responsible for the observed differences in PF4 levels, we examined the distribution of comorbidities among the subjects in each of the enrolled cohorts (Table 10). The only significant differences observed were that of a reduced incidence of cancer in the lipedema cohort when compared with those with lymphedema and increased hypertension and musculoskeletal disease in the control group when compared with those with lymphedema. Of note, no identified platelet disorder was listed among these patients. In parallel with the human clinical observations, PF4 was also upregulated in both young and old $\mathrm{Prox}^{+/-}$mice (Figure 2B) but not in $o b / o b$ mice. These findings suggest that PF4 could be a novel biomarker for lymphatic disorders.

Then, to explore whether PF4 can distinguish normal lean and obese human subjects (no symptomatic lymphatic malfunction) from those with lymphatic disorders, we further separated lean and obese normal and lymphatic-affected individuals. As shown in Figure 4, C and D, the PF4 level was not statistically different in lean or obese normal or affected subjects. This suggests that PF4 is a promising biomarker capable of distinguishing normal subjects from those with lymphatic defects independent of the presence or absence of obesity.

\section{Discussion}

Lymphedema is a devastating disease that lacks early diagnostic tools and readily available pharmacological interventions. Current accurate diagnosis of early or subclinical disease often relies upon sophisticated imaging techniques, which can be relatively invasive. Less invasive screening tools are not yet available. Although the more advanced stages of lymphedema can be clinically diagnosed, subtle, early, and subclinical disease can be elusive. Furthermore, with the recent surge of newly identified functional roles for the lymphatic vasculature in a variety of normal and pathological conditions (e.g., obesity, cardiovascular disease, and neurodegenerative disorders) (18-20), it is possible that individuals with any of those pathologies might be grossly asymptomatic for any of the typical features of lymphatic dysfunction. In such circumstances, the ready availability of reliable biomarkers could play a defining role in the screening and diagnosis of more subtle forms of lymphatic defects.

This analysis, using mouse models and individuals with a variety of lymphatic pathologies, identified PF4 as a promising diagnostic marker for lymphatic disorders. The levels of PF4 were increased in both young and old Prox $1^{+/-}$mice (before and after the onset of obesity), as well as in lymphedema, lipedema, and patients with heritable developmental diseases of the lymphatics. PF4, also called 
Table 3. Demographics and disease characterization of the lymphatic subjects and normal controls

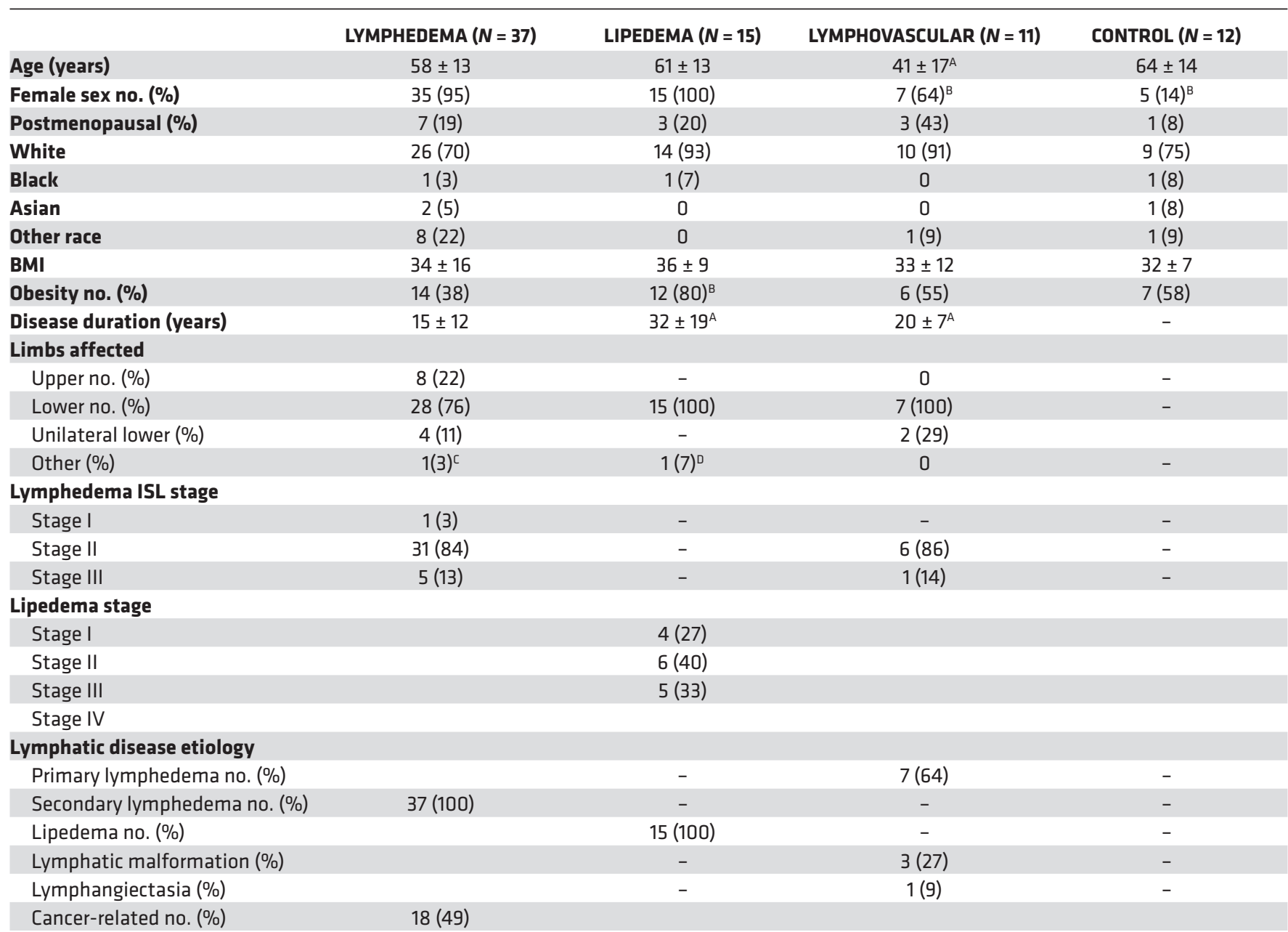

Kruskal-Wallis tests were performed for all continuous variables, and statistical comparisons were performed relative to lymphedema, unless indicated. Continuous variables were subjected to Mann-Whitney $U$ testing and discontinuous variables to the Fisher exact test. Lymphatic disease etiology was not statistically examined. Unless indicated, the subject populations did not differ statistically. ${ }^{A} P<0.01,{ }^{B} P<0.05$, compared with lymphedema subjects.

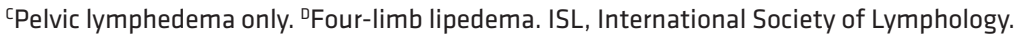

CXCL4, is a chemokine that is packaged in platelet $\alpha$-granules and is secreted upon activation during inflammation and wound healing. Although this study does not identify the cell of origin of exosomal $\mathrm{PF} 4$ or the mechanism underlying exosomal PF4 secretion, it is possible to speculate that structurally and functionally defective lymphatics are responsible for mediating such signaling. Besides regulating hemostasis and thrombosis, platelets play an important developmental role in the separation of the blood and lymphatic vascular networks $(43,44)$. Platelets are activated by lymphatic endothelial cells to form a plug at the level of the lymphovenous valve, the structure where the central lymphatic vasculature connects to the blood vascular system. In mice, the failure to form such a platelet plug results in the reflux of blood into the lymphatic vessels, and these mice develop lymphedema $(45,46)$. Interestingly, it has been reported that PF4 is also increased in a mouse model of acute surgical lymphedema detected by cDNA microarray analysis (47). Prior studies suggested that PF4 inhibits angiogenesis in vivo and in vitro $(48,49)$. For example, PF4 inhibits FGF2 and VEGF signaling through heparin-dependent and -independent mechanisms $(50,51)$. However, whether increased exosomal PF4 inhibits lymphangiogenesis in vivo is not clear.

A few lines of evidence support the hypothesis that PF4 might play a role in lymphedema. It has been shown that PF4 induces chemotaxis of T lymphocytes and upregulates T helper 2 (Th2) cytokines 
Table 4. List of increased proteins in patients with secondary lymphedema compared with healthy individuals

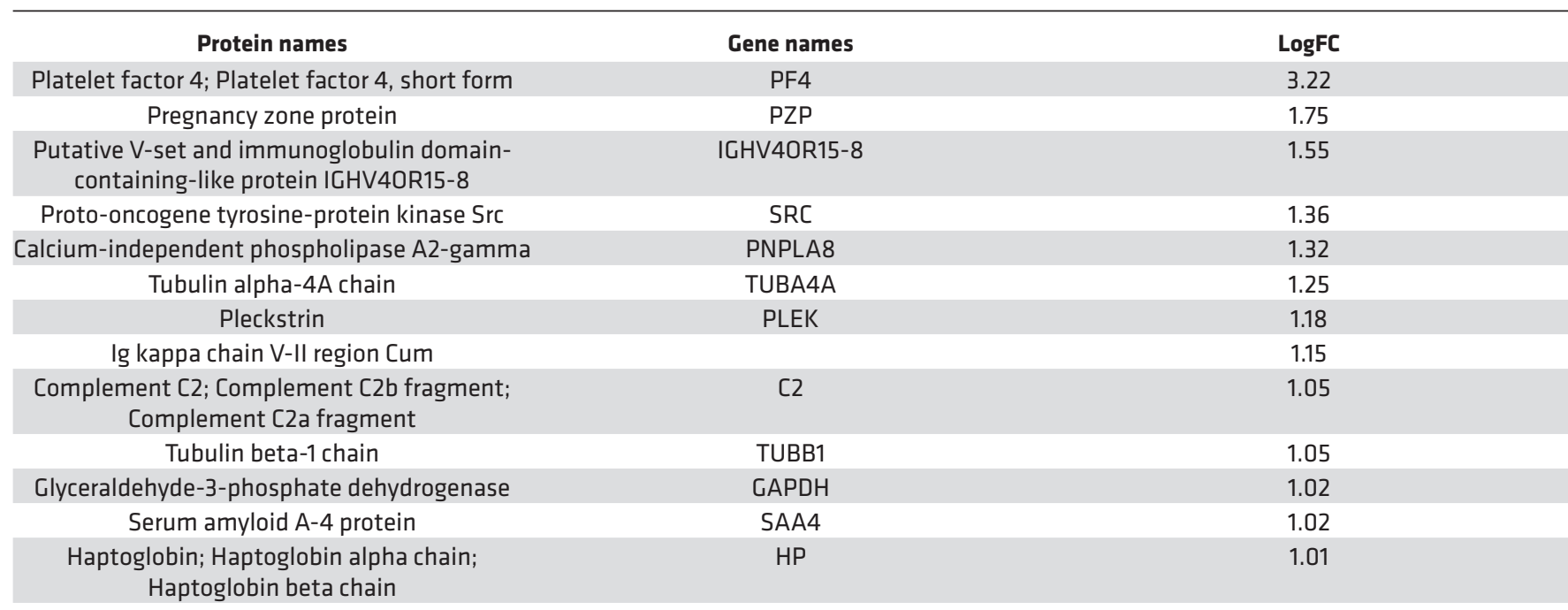

FC, fold change.

in a CXCR3-dependent manner $(52,53)$. This is relevant because T cells, including Th2 cells, are known to infiltrate lymphedematous tissue and play a role in inflammation, fibrosis, and lymphangiogenesis (54-56). Blocking Th2 differentiation decreases fibrosis, improves lymphatic function, and delays the progression of lymphedema (56). The elevated levels of PF4 detected in plasma-circulating exosomes of affected individuals might contribute to the recruitment and stimulation of Th2 cells in patients with lymphedema. Moreover, it has also been reported that blood plasma PF4 levels are increased in patients with Crohn's disease (57-63), a disorder that has been recently shown to feature lymphatic alterations $(42,64)$. The elevated platelet count appears to correlate with the presence of immature platelets in blood, which might play a role in predisposing patients with IBD to thrombus development. It is reasonable to speculate that the increased levels of PF4 in Crohn's disease could also, at least partially, be the consequence of the associated alterations in the mesenteric lymphatic vasculature. In addition, lipopolysaccharides (LPSs), which trigger proinflammatory responses in endothelial cells, increase PF4 levels and cell permeability by reducing tight junction proteins in cultured human umbilical vein endothelial

Table 5. List of decreased proteins in patients with secondary lymphedema compared with healthy individuals

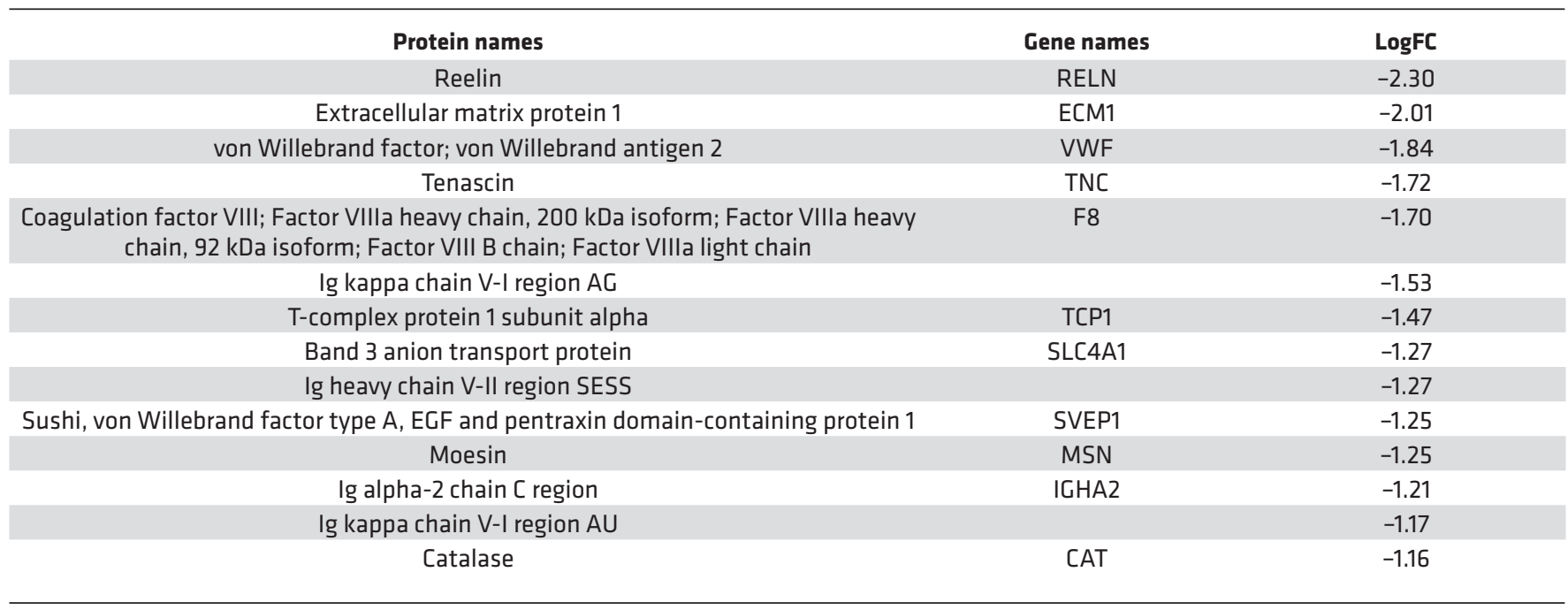


Table 6. List of increased proteins in patients with lymphovascular disease compared with healthy individuals

\begin{tabular}{|c|c|c|}
\hline Protein names & Gene names & LogFC \\
\hline Ig kappa chain V-IV region; Ig kappa chain V-IV region JI & IGKV4-1 & 8.14 \\
\hline Pregnancy zone protein & PZP & 3.46 \\
\hline Ig lambda chain V-V region DEL & & 2.51 \\
\hline Platelet factor 4; Platelet factor 4 , short form & PF4 & 2.41 \\
\hline C-reactive protein; C-reactive protein(1-205) & CRP & 2.27 \\
\hline Alcohol dehydrogenase class-3 & $\mathrm{ADH} 5$ & 1.99 \\
\hline Peroxiredoxin-2 & PRDX2 & 1.86 \\
\hline Desmoglein-1 & DSG1 & 1.81 \\
\hline Ig kappa chain V-III region $\mathrm{Ti}$ & & 1.80 \\
\hline Ig heavy chain V-III region TIL & & 1.79 \\
\hline Ig lambda chain V-VI region WLT; Ig lambda chain V-VI region EB4 & & 1.48 \\
\hline Complement factor $\mathrm{D}$ & CFD & 1.45 \\
\hline Proto-oncogene tyrosine-protein kinase Src & SRC & 1.34 \\
\hline Ig lambda chain V-II region BUR & & 1.32 \\
\hline Fibulin-5 & FBLN5 & 1.28 \\
\hline Clathrin heavy chain 1 & CLTC & 1.24 \\
\hline Ig kappa chain V-I region Wes & & 1.22 \\
\hline Ig lambda chain V-I region WAH & & 1.21 \\
\hline Ig lambda chain $\mathrm{V}$ region $4 \mathrm{~A}$ & & 1.20 \\
\hline Ig kappa chain V-II region TEW & & 1.20 \\
\hline Fetuin-B & FETUB & 1.19 \\
\hline Ig lambda chain V-I region NIG-64; Ig lambda chain V-I region BL2 & & 1.11 \\
\hline Ig lambda chain V-IV region MOL & & 1.10 \\
\hline Ig lambda chain V-I region NEW & & 1.06 \\
\hline Ig heavy chain V-III region HIL & & 1.02 \\
\hline
\end{tabular}

cells (65). The authors suggested that the effect of LPS on cell permeability is mediated by PF4 because it can be abolished by PF4 neutralizing antibodies, and PF4 itself decreases tight junction proteins and promotes cell permeability (65). It could be speculated that PF4 might increase blood vessel permeability and reduce lymphangiogenesis as contributing factors in lymphatic diseases. Supporting this hypothesis, pathological alterations of leukotriene biology have been observed in both murine and human lymphedema, with evidence of antilymphangiogenic concentrations of leukotriene $\mathrm{B}_{4}\left(\mathrm{LTB}_{4}\right)$ in these individuals (66). It is notable that $\mathrm{LTB}_{4}$ also induced endothelial cell permeability in vivo (67). Future investigations of the role of PF4 in lymphatic dysfunction should encompass exploration of the relationship of PF4 to leukotriene-mediated effects in the pathogenesis of lymphedema and lymphatic disorders.

Several studies have suggested a close association of excessive fat accumulation with lymphatic dysfunction. We have previously shown that $\operatorname{Prox}^{+/-}$mice with defective lymphatics develop adult-onset obesity, likely a consequence of chyle leakage $(18,68)$. Comparison of the exosome protein profile between $\mathrm{ProxI}^{+/-}$and $\mathrm{ob} / \mathrm{ob}$ mice demonstrated substantial differences, and, specifically, PF4 was not 
Table 7. List of top $\mathbf{5 0}$ decreased proteins in patients with lymphovascular disease compared with healthy individuals

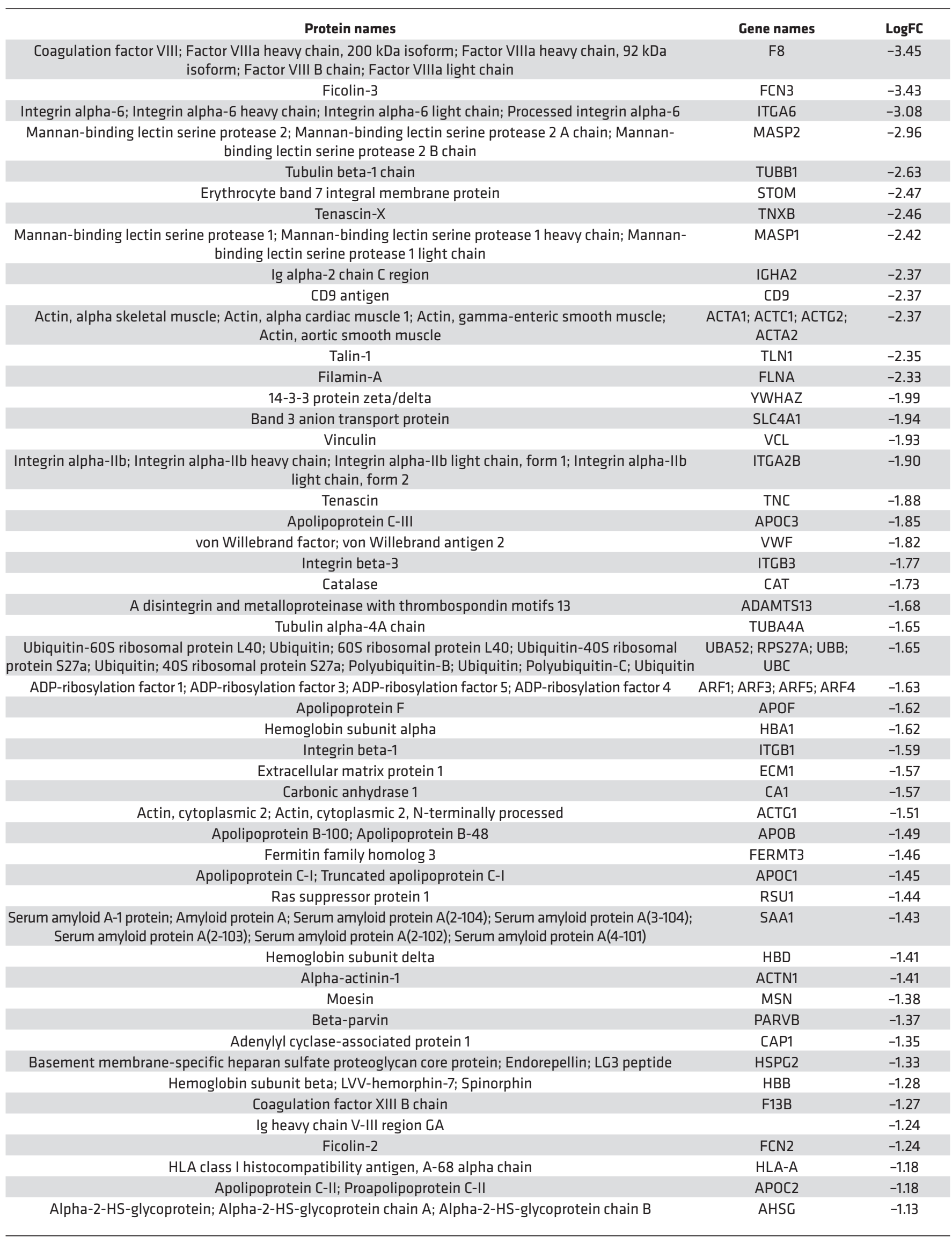


Table 8. List of increased proteins in patients with lipedema compared with healthy individuals

\begin{tabular}{|c|c|c|}
\hline Protein names & Gene names & LogFC \\
\hline Ig kappa chain V-IV region; Ig kappa chain V-IV region JI & IGKV4-1 & 7.29 \\
\hline Platelet glycoprotein Ib beta chain & GP1BB & 2.61 \\
\hline Pleckstrin & PLEK & 2.13 \\
\hline Myosin light polypeptide 6 & MYL6 & 2.12 \\
\hline Ig kappa chain V-III region IARC/BL41 & & 2.12 \\
\hline Tubulin alpha- $4 A$ chain & TUBA4A & 1.39 \\
\hline Alpha-actinin-1 & ACTN1 & 1.37 \\
\hline Transgelin-2 & TAGLN2 & 1.36 \\
\hline Platelet factor 4; Platelet factor 4, short form & PF4 & 1.26 \\
\hline $\begin{array}{l}\text { Actin, alpha skeletal muscle; Actin, alpha cardiac muscle 1; Actin, gamma-enteric smooth muscle; } \\
\text { Actin, aortic smooth muscle }\end{array}$ & $\begin{array}{l}\text { ACTA1; ACTC1; ACTC2; } \\
\text { ACTA2 }\end{array}$ & 1.25 \\
\hline Tropomyosin alpha-4 chain & TPM4 & 1.06 \\
\hline Complement factor $\mathrm{H}$-related protein 1 & CFHR1 & 1.04 \\
\hline $\begin{array}{l}\text { Complement C4-A; Complement C4 beta chain; Complement C4-A alpha chain; } C 4 \text { a anaphylatoxin; } \\
\text { C4b-A; C4d-A; Complement C4 gamma chain }\end{array}$ & C4A & 1.04 \\
\hline
\end{tabular}

increased in $o b / o b$ mice. These data suggest that PF4 levels might also be useful to identify obese individuals in which at least some of the underlying pathogenesis of excessive fat accumulation could be subtle and asymptomatic lymphatic leakage. Finally, our results support the prevailing hypothesis that in lipedema, lymphatic dysfunction plays a role in the pathogenesis of the disease, as has previously been suggested on the basis of imaging attributes (10-13).

Some questions to be addressed in future studies include: (i) as PF4 RNA levels are increased in the subacute tail wound model of acquired lymphedema (47), additional studies are needed to determine whether PF4 is increased in local tissue or circulating exosomes; (ii) in vivo lymphedema models using $\mathrm{PF}^{4^{--}}$mice or anti-PF4 antibodies combined with platelet transfusion should provide insight about potential therapeutic application of PF4; (iii) appropriately powered future clinical investigations of lymphatic disease cohorts are necessary to explore the relationship of PF4 levels with disease mechanisms and disease severity.

\section{Methods}

Mouse studies. The $o b / o b$ mice were obtained from The Jackson Laboratory (69). Prox $1^{+/-}$mice were generated and reported previously (70).

Exosome purification and characterization. Gently mixed blood with EDTA was centrifuged at $500 \mathrm{~g}$ for 10 minutes at $10^{\circ} \mathrm{C}$. Supernatant was centrifuged at $3000 \mathrm{~g}$ for 20 minutes at $10^{\circ} \mathrm{C}$. Plasma was centrifuged at $12,000 \mathrm{~g}$ for 20 minutes at $10^{\circ} \mathrm{C}$ to remove microvesicles, and the supernatant was centrifuged at $100,000 \mathrm{~g}$ for 70 minutes at $10^{\circ} \mathrm{C}$. The exosomes in the pellet fraction were washed with $20 \mathrm{~mL}$ of PBS and centrifuged at $100,000 \mathrm{~g}$ for 70 minutes at $10^{\circ} \mathrm{C}$. The final exosome pellet was resuspended in $100 \mu \mathrm{L}$ of PBS for analysis.

Human studies. We recruited study subjects from the patient population of the Stanford Center for Lymphatic and Venous Disorders. The Administrative Panels for the Protection of Human Subjects of Stanford University (IRB 0000350) approved the protocols. Investigations were conducted according to the Declaration of Helsinki principles. Written consent was obtained from all recipients before inclusion in the studies. Phlebotomy was performed in the standard fashion, using a small-gauge needle inserted into the brachiocephalic vein; $30 \mathrm{cc}$ of blood was withdrawn in EDTA tubes, and the plasma was frozen at $-80^{\circ} \mathrm{C}$ for subsequent molecular analysis.

In order to be eligible for enrollment in this study, subjects were screened for the presence of lymphedema (primary or secondary), lipedema, and lymphatic malformations. The diagnosis of lymphedema was 
Table 9. List of decreased proteins in patients with lipedema compared with healthy individuals

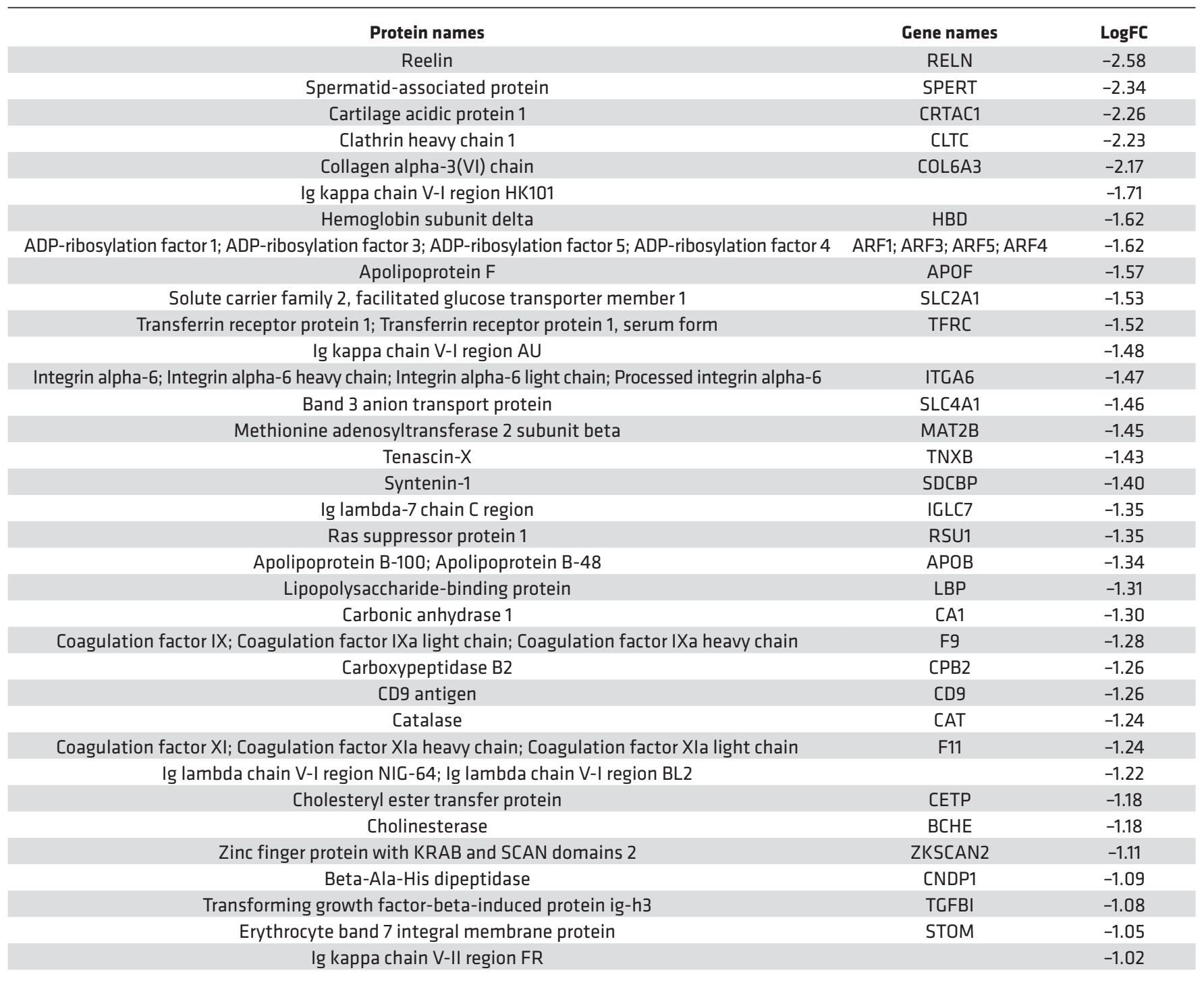

based upon clinical evaluation, using the criteria the International Society of Lymphology established. The diagnosis of lipedema is based on commonly accepted clinical attributes (71). Normal control subjects were recruited from the same cardiovascular clinic as those with lymphatic pathologies; eligibility for enrollment included the absence of any clinically identifiable lymphatic pathology and the willingness to participate. In each subject cohort, the presence of obesity was defined as BMI $>30$.

Mouse proteomic analysis. Proteins were dissolved using $8 \mathrm{M}$ urea in $100 \mathrm{mM}$ ammonium bicarbonate and $10 \mathrm{mM}$ DTT. After reduction, cysteines were alkylated in $30 \mathrm{mM}$ iodoacetamide. Proteins were then in solution and digested with Lys-C (endopeptidase Lys-C, Wako Chemicals) in $4 \mathrm{M}$ urea, followed by trypsinization (Trypsin Gold, Promega) in $2 \mathrm{M}$ urea. Digestions were stopped by adding trifluoroacetic acid, and the digests were desalted using $\mathrm{C}_{18}$ stage tips.

Samples were analyzed by liquid chromatography-tandem MS (LC-MS/MS) (Dionex 3000 coupled to Q-Exactive, Thermo Fisher Scientific). Peptides were separated by $\mathrm{C}_{18}$ chromatography (inner diameter of $75 \mu \mathrm{m} / 3 \mu \mathrm{m}$ particles, Nikkyo Technologies) using a gradient increasing from $1 \%$ B to $45 \%$ $\mathrm{B}$ in 135 minutes (A: $0.1 \%$ formic acid, B: acetonitrile in $0.1 \%$ formic acid). The peptides were electrosprayed $(3.4 \mathrm{kV})$ into the mass spectrometer through a heated capillary at $320^{\circ} \mathrm{C}$ and an S-Lens radio frequency (RF) level of $60 \%$. The mass spectrometer was operated in a data-dependent mode, with 


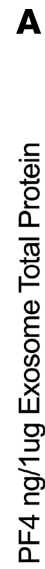

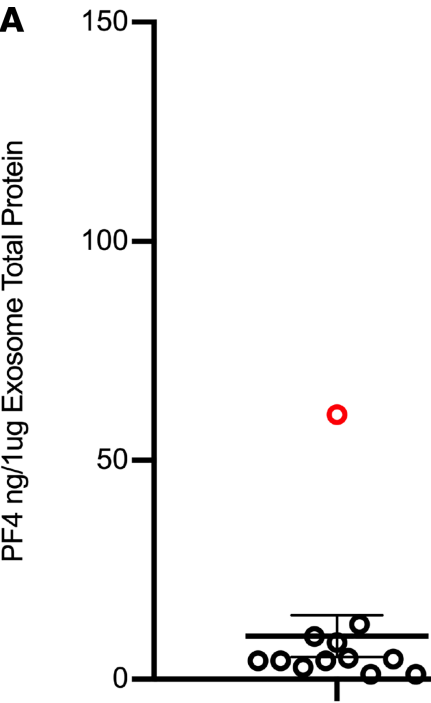

Control

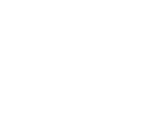

口

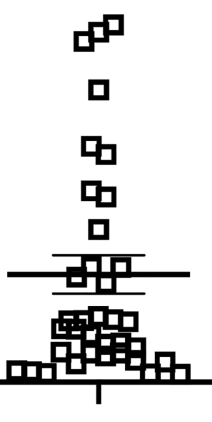

Secondary

Lymphedema

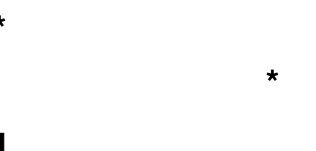

$\Delta$

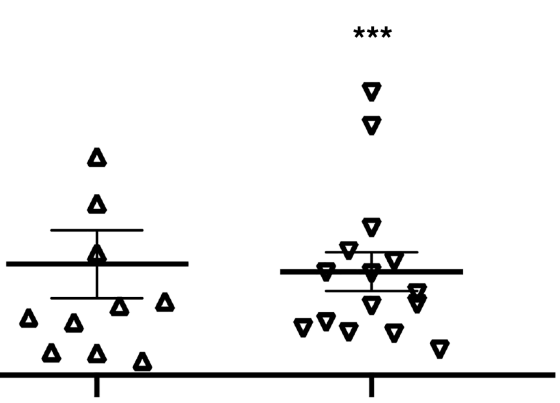

Lymphovascular Disease

Lipedema

B

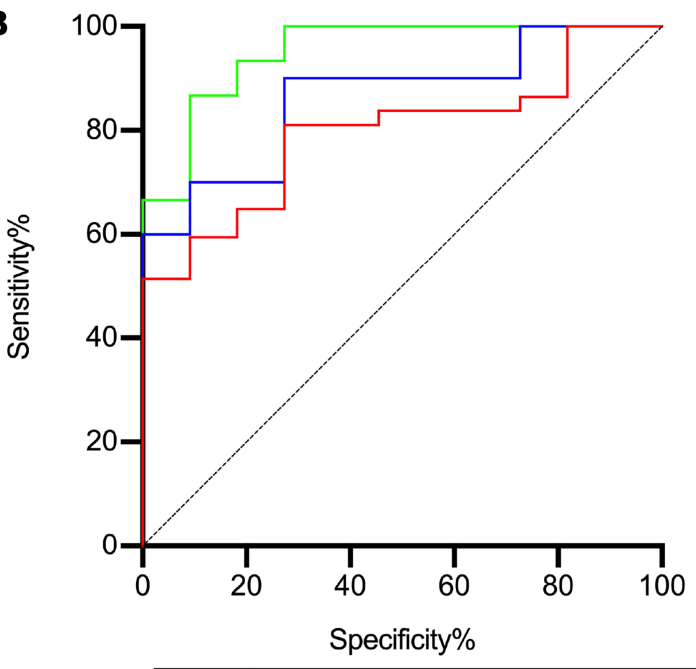

\begin{tabular}{|c|c|c|c|c|}
\hline & Cutpoint & Sensitivity\% & Specificity\% & AUC (95\% Cl) \\
\hline Secondary Lymphedema & $>10.24$ & 59.46 & 90.91 & $0.80(0.67$ to 0.93) \\
\hline Lymphovascular disease & $>10.93$ & 70.00 & 90.91 & $0.86(0.70$ to 1.00$)$ \\
Lipedema & $>9.71$ & 86.67 & 90.91 & $0.95(0.99$ to 1.00$)$ \\
\hline
\end{tabular}

C

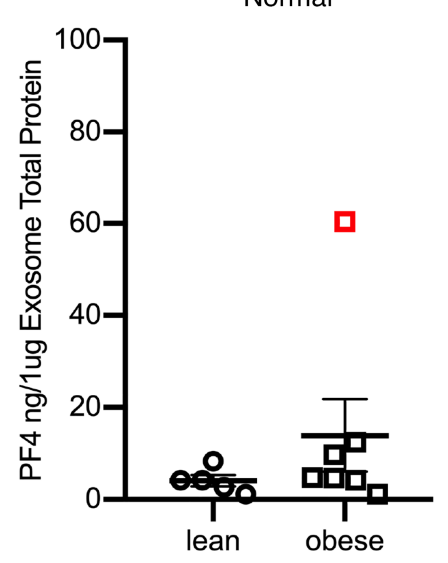

D

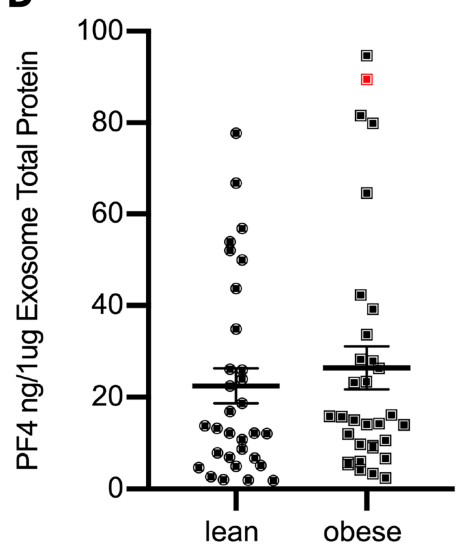

Figure 4. Validation of PF4 levels in plasma exosomes from individuals with normal lymphatics and patients with lymphatic disorders. (A) ELISA quantification of PF4 levels in exosomes from control subjects and indicated groups of patients. PF4 levels were normalized to the exosome protein content. (Red symbols indicate outliers detected by iterative Grubb's test and excluded from the statistical analysis. ${ }^{*}$ indicates $P \leq 0.05$,

${ }^{* *}$ indicates $P \leq 0.01$, and ${ }^{* * *}$ indicates $P \leq 0.001$ compared with control.) (B) ROC curve of PF4 for each diagnosis. The cutoff value of PF4 with sensitivity and specificity, as well as AUC and $\mathrm{Cl}$, are presented in a separate table. (AUC, area under the ROC curve; $\mathrm{Cl}$, confidence interval.) (C) The PF4 from individuals with normal lymphatics are further divided based on BMI $>30$, and the PF4 level from lean and obese individuals with normal lymphatics is not statisticalIy different (red symbol indicates the outlier in Figure 4A normal group detected by iterative Grubb's test group and excluded from the statistical analysis). (D) The PF4 from individuals with secondary lymphedema, lymphovascular disease, and lipedema are grouped into lean and obese based on BMI of 30 , and the PF4 level from lean and obese individuals with lymphatic disorders is not statistically different (red dot indicates the outlier in Figure 4A lymphovascular disease group detected by iterative Grubb's test group and are excluded from the statistical analysis). 
Table 10. Comorbidities (all in \%)

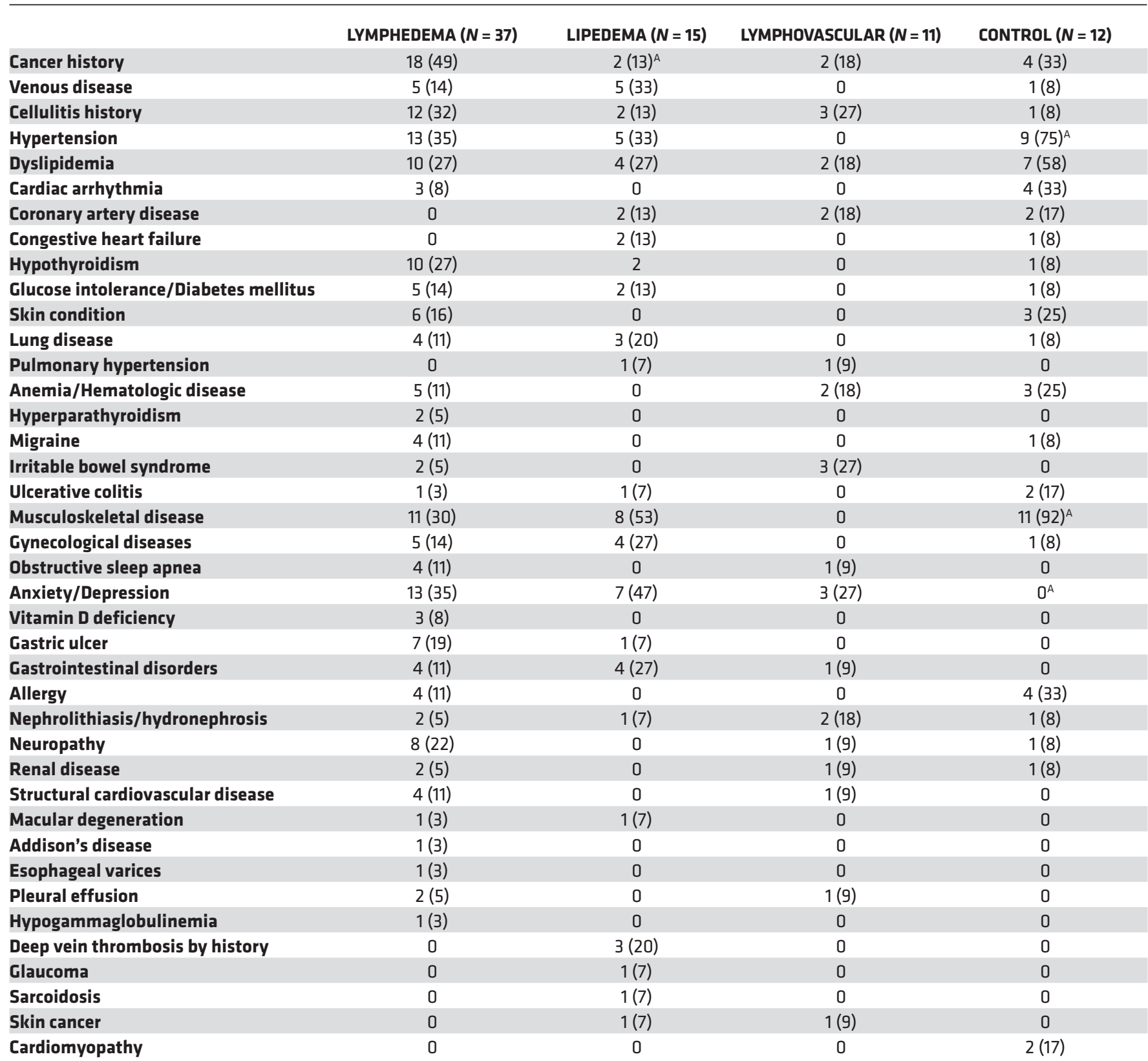

All statistical comparisons were with lymphedema and used the Fisher exact test. Unless designated, there were no statistically significant differences. ${ }^{A} P<0.05$ compared with lymphedema.

an automatic switch between the MS and MS/MS scans using a top 20 method (minimum automatic gain control target 3E3) and a dynamic exclusion time of 45 seconds. MS (300-1400 $\mathrm{m} / \mathrm{z}$ ) and MS/MS spectra were acquired with a resolution of 70,000 and 17,500 full width at half maximum (FWHM) $(200 \mathrm{~m} / z)$, respectively. Peptides were isolated using a 2 Thomson window and fragmented using higherenergy collisional dissociation at $27 \%$ normalized collision energy. The ion target values were $5 \mathrm{E} 5$ for MS (100-ms maximum injection time) and 2E5 for MS/MS (60-ms maximum injection time).

Raw files were processed with MaxQuant ( $\mathrm{v}$ 1.5.1.2) using the standard settings against a mouse protein database (UniProtKB/Swiss-Prot/TrEMBL, 43,539 sequences) supplemented with contaminants. Carbamidomethylation of cysteines was set as a fixed modification with oxidation of methionine and protein $\mathrm{N}$-term acetylation as variable modifications. Minimal peptide length was set to 7 
amino acids, and a maximum of 2 tryptic missed cleavages were allowed. Results were filtered at 0.01 FDR (peptide and protein level). An arbitrary criterion of fold change $>0.5$ or $<-0.5$ was used to define proteins as upregulated or downregulated.

Human proteomic analysis. Proteins were dissolved using $8 \mathrm{M}$ urea in $100 \mathrm{mM}$ Tris- $\mathrm{HCl} \mathrm{pH}$ 8.0. Protein concentration was determined using the Pierce $660 \mathrm{~nm}$ Protein Assay (Bio-Rad) using BSA as standard. Then, samples $(10-20 \mu \mathrm{g})$ were digested by means of the standard filter aided sample preparation protocol. Briefly, proteins were reduced and alkylated [15 mM tris-2(-carboxyethyl)-phosphine, $30 \mathrm{mM}$ chloroacetamide, 30 minutes in the dark, room temperature] and sequentially digested with Lys-C (Wako) (protein/ enzyme ratio 1:50, overnight at room temperature) and trypsin (Promega) (protein/enzyme ratio 1:100, 6 hours at $37^{\circ} \mathrm{C}$ ). Resulting peptides were desalted using $\mathrm{C}_{18}$ stage tips.

LC-MS/MS was done by coupling a nanoLC-Ultra 1D+ system (Eksigent) to an LTQ Orbitrap Velos mass spectrometer (Thermo Fisher Scientific) via a Nanospray Flex source (Thermo Fisher Scientific). Peptides were loaded into a trap column (NS-MP-10 BioSphere C18 $5 \mu \mathrm{m}, 20 \mathrm{~mm}$ length, Nanoseparations) for 10 minutes at a flow rate of $2.5 \mu \mathrm{L} / \mathrm{min}$ in $0.1 \%$ formic acid (FA). Then peptides were transferred to an analytical column (ReproSil Pur C18-AQ $1.9 \mu \mathrm{m}, 400 \mathrm{~mm}$ length and $0.075 \mathrm{~mm}$ ID) and separated using a 150-minute linear gradient (buffer A: 4\% acetonitrile [ACN], 0.1\% FA; buffer B: 100\% $\mathrm{ACN}, 0.1 \% \mathrm{FA}$ ) at a flow rate of $250 \mathrm{~nL} / \mathrm{min}$. The gradient used was: $0-2$ minutes $2 \% \mathrm{~B}, 3-133$ minutes $30 \% \mathrm{~B}, 134-144$ minutes $98 \% \mathrm{~B}$, and $145-150$ minutes $2 \% \mathrm{~B}$. The peptides were electrosprayed $(1.8 \mathrm{kV})$ into the mass spectrometer with a PicoTip emitter (360/20 Tube OD/ID $\mu \mathrm{m}$, tip ID $10 \mu \mathrm{m})(\mathrm{New}$ Objective), a heated capillary temperature of $325^{\circ} \mathrm{C}$, and an S-Lens RF level of $60 \%$. The mass spectrometer was operated in a data-dependent mode, with an automatic switch between MS and MS/MS scans using a top 20 method (threshold signal $\geq 800$ counts and dynamic exclusion of 45 seconds). MS spectra (350$1500 \mathrm{~m} / z)$ were acquired in the Orbitrap with a resolution of 60,000 FWHM $(400 \mathrm{~m} / z)$. Peptides were isolated using a $1.5 \mathrm{Th}$ window and fragmented using collision-induced dissociation with linear ion trap readout at a normalized collision energy of 35\% ( $0.25 \mathrm{Q}$ value and 10-ms activation time). The ion target values were $1 \mathrm{E} 6$ for MS (500-ms max injection time) and 5000 for MS/MS (100-ms max injection time).

Raw files were processed with MaxQuant ( $\mathrm{v}$ 1.5.3.30) using the standard settings against a human protein database (UniProtKB/Swiss-Prot, December 2013, 20,584 sequences) supplemented with contaminants. Carbamidomethylation of cysteines was set as a fixed modification with oxidation of methionine and protein $\mathrm{N}$-term acetylation as variable modifications. Minimal peptide length was set to 7 amino acids, and a maximum of 2 tryptic missed cleavages were allowed. Results were filtered at 0.01 FDR (peptide and protein level). Afterward, the "proteinGroups.txt" file was loaded in Prostar (v1.18) (72) using the intensity values for further statistical analysis. Briefly, proteins with fewer than 4 valid values in at least 1 experimental condition were filtered out. Then, a global normalization of $\log _{2}$-transformed intensities across samples was performed using the LOESS function. Missing values were imputed using the algorithms SLSA (73) for partially observed values and DetQuantile for values missing on an entire condition. Differential analysis was done using the empirical Bayes statistics Limma package. Proteins with a $P$ value less than 0.1 and a $\log _{2}$ ratio greater than 1 or less than -1 were defined as regulated. The FDR was estimated to be up to $10 \%$ by Benjamini-Hochberg.

ELISA. Total protein quantification in exosomes was performed using the BCA protein assay kit (Pierce, Thermo Fisher Scientific). PF4 concentration in exosomes was quantified using a human PF4 ELISA kit (R\&D Systems, Bio-Techne) according to the manufacturer's instructions. The standards and the samples were run in duplicate. The results were read using a Synergy 2 plate reader (BioTek, Agilent). PF4 concentration was normalized to total protein content in exosomes.

Statistics. The analysis method for MS, demographics, and comorbidities is detailed in the text and table legends. All statistical analyses for ELISA were performed using GraphPad Prism 8.0. Data with parametric distribution were analyzed using unpaired 2-tailed Student's $t$ tests or 1-way analysis of variance; data with nonparametric distribution were analyzed by the Kruskal-Wallis test unless specified in the legends. All analyses with $P$ values below 0.05 were considered statistically significant. Data represent mean \pm SEM.

Study approval. All the mouse work was approved by the Northwestern University Animal Care and IACUC guidelines. Study subjects were recruited from the patient population of the Stanford Center for Lymphatic and Venous Disorders. The Administrative Panels for the Protection of Human Subjects of Stanford University (IRB 0000350) approved the protocols. Investigations were conducted according to the Declaration of Helsinki principles. Written informed consent was obtained from all participants before inclusion in the studies. 


\section{Author contributions}

WM and GO designed the experiments. WM conducted the experiments and acquired and analyzed the data. HJG and NE conducted experiments and acquired data. SGR provided patients' samples and performed the demographic and comorbidity analysis. PXE, ABM, and JM conducted MS experiments and analysis. WM, HP, SGR, and GO wrote the manuscript.

\section{Acknowledgments}

This work was partially supported by the NIH (grant R01HL073402 to GO). WM was partially supported by NIH T32 HL134633 and NE by CONICYT/FONDECYT N¹204562. Additional support came from the European Union Horizon 2020 program INFRAIA project Epic-XS (project 823839) to HP.

Address correspondence to: Guillermo Oliver, Feinberg Cardiovascular and Renal Research Institute, Simpson-Querrey Biomedical Research Center, 303 East Superior Street, 8-519, Chicago, Illinois 60611, USA. Phone: 312.503.1651; Email: guillermo.oliver@northwestern.edu.

HJG's present address is: Department of Surgery, St. Jude Children's Research Hospital, Memphis, Tennessee, USA. NE's present address is: Lymphatic Vasculature and Inflammation Research Laboratory, Facultad de Ciencias de la Salud, Instituto de Ciencias Biomédicas, Universidad Autónoma de Chile, Talca, Chile.

1. Escobedo N, Oliver G. Lymphangiogenesis: origin, specification, and cell fate determination. Annu Rev Cell Dev Biol. 2016;32:677-691.

2. Rockson SG. Lymphedema. Am J Med. 2001;110(4):288-295.

3. Witte CL. Pumps and lymphedema. Lymphology. 2001;34(4):150-151.

4. Greene AK, Maclellan RA. Obesity-induced upper extremity lymphedema. Plast Reconstr Surg Glob Open. 2013;1(7):e59.

5. Wang Y, Oliver G. Current views on the function of the lymphatic vasculature in health and disease. Genes Dev. 2010;24(19):2115-2126.

6. Tavakkolizadeh A, Wolfe KQ, Kangesu L. Cutaneous lymphatic malformation with secondary fat hypertrophy. Br J Plast Surg. 2001;54(4):367-369.

7. Schirger A, Harrison EG, Janes JM. Idiopathic lymphedema. Review of 131 cases. JAMA. 1962;182:14-22.

8. Pond CM. Adipose tissue and the immune system. Prostaglandins Leukot Essent Fatty Acids. 2005;73(1):17-30.

9. Rosen ED. The molecular control of adipogenesis, with special reference to lymphatic pathology. Ann N Y Acad Sci. 2002;979:143-58.

10. Lohrmann C, Foeldi E, Langer M. MR imaging of the lymphatic system in patients with lipedema and lipo-lymphedema. Microvasc Res. 2009;77(3):335-339.

11. Bilancini S, Lucchi M, Tucci S, Eleuteri P. Functional lymphatic alterations in patients suffering from lipedema. Angiology. 1995;46(4):333-339.

12. Forner-Cordero I, Oliván-Sasot P, Ruiz-Llorca C, Muñoz-Langa J. Lymphoscintigraphic findings in patients with lipedema. Rev Esp Med Nucl Imagen Mol. 2018;37(6):341-348.

13. Boursier V, Pecking A, Vignes S. [Comparative analysis of lymphoscintigraphy between lipedema and lower limb lymphedema]. J Mal Vasc. 2004;29(5):257-261.

14. Wold LE, Hines EA, Allen EV. Lipedema of the legs; a syndrome characterized by fat legs and edema. Ann Intern Med. 1951;34(5):1243-1250

15. Okhovat JP, Alavi A. Lipedema: a review of the literature. Int J Low Extrem Wounds. 2015;14(3):262-267.

16. Shin BW, Sim YJ, Jeong HJ, Kim GC. Lipedema, a rare disease. Ann Rehabil Med. 2011;35(6):922-927.

17. Wagner S. Lymphedema and lipedema - an overview of conservative treatment. VASA. 2011;40(4):271-279.

18. Harvey NL, et al. Lymphatic vascular defects promoted by Prox1 haploinsufficiency cause adult-onset obesity. Nat Genet. 2005;37(10):1072-1081.

19. Escobedo N, Oliver G. The lymphatic vasculature: its role in adipose metabolism and obesity. Cell Metab. 2017;26(4):598-609.

20. Petrova TV, Koh GY. Organ-specific lymphatic vasculature: from development to pathophysiology. J Exp Med. 2018;215(1):35-49.

21. Lin J, et al. Exosomes: novel biomarkers for clinical diagnosis. ScientificWorldJournal. 2015;2015:657086.

22. Colombo M, Raposo G, Théry C. Biogenesis, secretion, and intercellular interactions of exosomes and other extracellular vesicles. Annu Rev Cell Dev Biol. 2014;30:255-289.

23. Raposo G, Stoorvogel W. Extracellular vesicles: exosomes, microvesicles, and friends. J Cell Biol. 2013;200(4):373-383

24. van Balkom BW, Eisele AS, Pegtel DM, Bervoets S, Verhaar MC. Quantitative and qualitative analysis of small RNAs in human endothelial cells and exosomes provides insights into localized RNA processing, degradation and sorting. J Extracell Vesicles. $2015 ; 4: 26760$

25. Thakur BK, et al. Double-stranded DNA in exosomes: a novel biomarker in cancer detection. Cell Res. 2014;24(6):766-769.

26. van der Pol E, Böing AN, Harrison P, Sturk A, Nieuwland R. Classification, functions, and clinical relevance of extracellular vesicles. Pharmacol Rev. 2012;64(3):676-705.

27. Valadi H, Ekström K, Bossios A, Sjöstrand M, Lee JJ, Lötvall JO. Exosome-mediated transfer of mRNAs and microRNAs is a novel mechanism of genetic exchange between cells. Nat Cell Biol. 2007;9(6):654-659.

28. Taylor DD, Gercel-Taylor C. The origin, function, and diagnostic potential of RNA within extracellular vesicles present in 
human biological fluids. Front Genet. 2013;4:142.

29. Kahlert C, et al. Identification of double-stranded genomic DNA spanning all chromosomes with mutated KRAS and p53 DNA in the serum exosomes of patients with pancreatic cancer. J Biol Chem. 2014;289(7):3869-3875.

30. Waldenström A, Gennebäck N, Hellman U, Ronquist G. Cardiomyocyte microvesicles contain DNA/RNA and convey biological messages to target cells. PLoS One. 2012;7(4):e34653.

31. Lindström P. The physiology of obese-hyperglycemic mice [ob/ob mice]. ScientificWorldJournal. 2007;7:666-685.

32. Zhang Y, Proenca R, Maffei M, Barone M, Leopold L, Friedman JM. Positional cloning of the mouse obese gene and its human homologue. Nature. 1994;372(6505):425-432.

33. Friedman JM, Halaas JL. Leptin and the regulation of body weight in mammals. Nature. 1998;395(6704):763-770.

34. Filipe V, Hawe A, Jiskoot W. Critical evaluation of Nanoparticle Tracking Analysis (NTA) by NanoSight for the measurement of nanoparticles and protein aggregates. Pharm Res. 2010;27(5):796-810.

35. Slungaard A. Platelet factor 4: a chemokine enigma. Int J Biochem Cell Biol. 2005;37(6):1162-1167.

36. Kowalska MA, Rauova L, Poncz M. Role of the platelet chemokine platelet factor 4 (PF4) in hemostasis and thrombosis. Thromb Res. 2010;125(4):292-296.

37. Sachais BS, Higazi AA, Cines DB, Poncz M, Kowalska MA. Interactions of platelet factor 4 with the vessel wall. Semin Thromb Hemost. 2004;30(3):351-358.

38. Bakogiannis C, Sachse M, Stamatelopoulos K, Stellos K. Platelet-derived chemokines in inflammation and atherosclerosis. Cytokine. 2019;122:154157.

39. Grubbs FE. Sample criteria for testing outlying observations. Ann Math Stat. 1950;21(1):27-58.

40. Shen W, Li Y, Cao L, Cai X, Ge Y, Zhu W. Decreased expression of Prox1 is associated with postoperative recurrence in Crohn's disease. J Crohns Colitis. 2018;12(10):1210-1218.

41. Van Kruiningen HJ, Hayes AW, Colombel JF. Granulomas obstruct lymphatics in all layers of the intestine in Crohn's disease. APMIS. 2014;122(11):1125-1129.

42. Heatley RV, Bolton PM, Hughes LE, Owen EW. Mesenteric lymphatic obstruction in Crohn's disease. Digestion. 1980;20(5):307-313.

43. Carramolino L, Fuentes J, García-Andrés C, Azcoitia V, Riethmacher D, Torres M. Platelets play an essential role in separating the blood and lymphatic vasculatures during embryonic angiogenesis. Circ Res. 2010;106(7):1197-1201.

44. Osada M, et al. Platelet activation receptor CLEC-2 regulates blood/lymphatic vessel separation by inhibiting proliferation, migration, and tube formation of lymphatic endothelial cells. J Biol Chem. 2012;287(26):22241-22252.

45. Welsh JD, Kahn ML, Sweet DT. Lymphovenous hemostasis and the role of platelets in regulating lymphatic flow and lymphatic vessel maturation. Blood. 2016;128(9):1169-1173.

46. Hess PR, et al. Platelets mediate lymphovenous hemostasis to maintain blood-lymphatic separation throughout life. J Clin Invest. 2014;124(1):273-284.

47. Tabibiazar R, et al. Inflammatory manifestations of experimental lymphatic insufficiency. PLoS Med. 2006;3(7):e254.

48. Maione TE, et al. Inhibition of angiogenesis by recombinant human platelet factor- 4 and related peptides. Science. 1990;247(4938):77-79.

49. Tanaka T, Manome Y, Wen P, Kufe DW, Fine HA. Viral vector-mediated transduction of a modified platelet factor $4 \mathrm{cDNA}$ inhibits angiogenesis and tumor growth. Nat Med. 1997;3(4):437-442.

50. Jouan V, et al. Inhibition of in vitro angiogenesis by platelet factor-4-derived peptides and mechanism of action. Blood. 1999;94(3):984-993.

51. Lord MS, Cheng B, Farrugia BL, McCarthy S, Whitelock JM. Platelet factor 4 binds to vascular proteoglycans and controls both growth factor activities and platelet activation. J Biol Chem. 2017;292(10):4054-4063.

52. Mueller A, et al. CXCL4-induced migration of activated T lymphocytes is mediated by the chemokine receptor CXCR3. J Leukoc Biol. 2008;83(4):875-882.

53. Romagnani P, et al. CXCR3-mediated opposite effects of CXCL10 and CXCL4 on TH1 or TH2 cytokine production. J Allergy Clin Immunol. 2005;116(6):1372-1379.

54. Zampell JC, Yan A, Elhadad S, Avraham T, Weitman E, Mehrara BJ. CD4(+) cells regulate fibrosis and lymphangiogenesis in response to lymphatic fluid stasis. PLoS One. 2012;7(11):e49940.

55. Ogata F, et al. Excess lymphangiogenesis cooperatively induced by macrophages and CD4(+) T cells drives the pathogenesis of lymphedema. J Invest Dermatol. 2016;136(3):706-714.

56. Avraham T, et al. Th2 differentiation is necessary for soft tissue fibrosis and lymphatic dysfunction resulting from lymphedema. FASEB J. 2013;27(3):1114-1126

57. Ye L, Zhang YP, Yu N, Jia YX, Wan SJ, Wang FY. Serum platelet factor 4 is a reliable activity parameter in adult patients with inflammatory bowel disease: a pilot study. Medicine (Baltimore). 2017;96(11):e6323.

58. Meuwis MA, et al. Proteomics for prediction and characterization of response to infliximab in Crohn's disease: a pilot study Clin Biochem. 2008;41(12):960-967.

59. Meuwis MA, et al. Biomarker discovery for inflammatory bowel disease, using proteomic serum profiling. Biochem Pharmacol. 2007;73(9):1422-1433.

60. Simi M, Leardi S, Tebano MT, Castelli M, Costantini FM, Speranza V. Raised plasma concentrations of platelet factor 4 (PF4) in Crohn's disease. Gut. 1987;28(3):336-338.

61. Yoshida H, Yilmaz CE, Granger DN. Role of tumor necrosis factor- $\alpha$ in the extraintestinal thrombosis associated with colonic inflammation. Inflamm Bowel Dis. 2011;17(11):2217-2223.

62. Danese S, Motte Cd Cde L, Fiocchi C. Platelets in inflammatory bowel disease: clinical, pathogenic, and therapeutic implications. Am J Gastroenterol. 2004;99(5):938-945.

63. Harries AD, Fitzsimons E, Fifield R, Dew MJ, Rhoades J. Platelet count: a simple measure of activity in Crohn's disease. Br Med J (Clin Res Ed). 1983;286(6376): 1476.

64. Kovi J, Duong HD, Hoang CT. Ultrastructure of intestinal lymphatics in Crohn's disease. Am J Clin Pathol. 1981;76(4):385-394.

65. Wang X, et al. Effects of CXCL4/CXCR3 on the lipopolysaccharide-induced injury in human umbilical vein endothelial cells. 
J Cell Physiol. 2019;234(12):22378-22385.

66. Tian W, et al. Leukotriene $\mathrm{B}_{4}$ antagonism ameliorates experimental lymphedema. Sci Transl Med. 2017;9(389):eaal3920.

67. Di Gennaro A, Kenne E, Wan M, Soehnlein O, Lindbom L, Haeggström JZ. Leukotriene B4-induced changes in vascular permeability are mediated by neutrophil release of heparin-binding protein (HBP/CAP37/azurocidin). FASEB J. 2009;23(6):1750-1757.

68. Escobedo N, et al. Restoration of lymphatic function rescues obesity in Prox1-haploinsufficient mice. JCI Insight. 2016;1(2):e85096.

69. Ewart-Toland A, Mounzih K, Qiu J, Chehab FF. Effect of the genetic background on the reproduction of leptin-deficient obese mice. Endocrinology. 1999;140(2):732-738.

70. Wigle JT, Oliver G. Prox1 function is required for the development of the murine lymphatic system. Cell. 1999;98(6):769-778.

71. Child AH, et al. Lipedema: an inherited condition. Am J Med Genet A. 2010;152A(4):970-976.

72. Wieczorek S, et al. DAPAR \& ProStaR: software to perform statistical analyses in quantitative discovery proteomics. Bioinformatics. 2017;33(1):135-136.

73. Bø TH, Dysvik B, Jonassen I. LSimpute: accurate estimation of missing values in microarray data with least squares methods Nucleic Acids Res. 2004;32(3):e34. 